RELATO DO CASO

\title{
Embolia atrial de trombo flutuante da veia safena magna após escleroterapia com microespuma ecoguiada
}

\section{Atrial embolism of floating thrombus of the great saphenous vein after microfoam ultrasound- guided sclerotherapy}

Rubens Pierry Ferreira Lopes', Robson Barbosa de Miranda², Celso Higutchi ${ }^{3}$, Tiago Calheiros de Holanda Barbosa4, Ohannes Kafejian ${ }^{5}$

\begin{abstract}
Resumo
A escleroterapia com microespuma vem sendo utilizada amplamente no tratamento da doença venosa varicosa. No entanto, a despeito da sua pouca invasividade e segurança, complicações potencialmente graves e letais já foram descritas, como o acidente vascular cerebral e parada cardiorrespiratória. Descrevemos um caso de embolia atrial tardia de trombo flutuante da junção safeno-femoral de veia safena magna varicosa, após escleroterapia com microespuma de polidocanol guiada por ultrassom, e o tratamento dessa complicação.
\end{abstract}

Palavras-chave: Embolia; tromboembolia; escleroterapia; ultrassonografia doppler; veia safena.

\begin{abstract} treatment of this complication.

\section{Introdução}

A escleroterapia com microespuma (EM) representa uma abordagem terapêutica cada vez mais utilizada para a insuficiência venosa crônica (IVC), principalmente por ser efetiva, segura e menos invasiva. No entanto, podem ocorrer complicações graves e ameaçadoras da vida. Este relato descreve um caso de embolia atrial tardia após EM guiada por ultrassom de veia safena magna varicosa.
\end{abstract}

Microfoam sclerotherapy has been widely used in the treatment of varicose vein disease. However, despite its low invasiveness and safety, potentially serious and lethal complications have been described, such as stroke and cardiorespiratory arrest. We describe a case of delayed atrial embolism of floating thrombus in the saphenofemoral junction of a great saphenous varicose vein after microfoam ultrasound-guided sclerotherapy, as well as the

Keywords: Embolism; thromboembolism; sclerotherapy; ultrasonography; doppler; saphenous vein.

\section{Relato do caso}

Indicou-se, em regime ambulatorial, a EM em um homem de 67 anos com IVC sintomática classe funcional CEAP C5 no membro inferior direito (MID). Sua história médica incluía hipertensão arterial e insuficiência cardíaca (IC) grau II da classificação NYHA. Os estudos hematológicos, da coagulação e a radiografia torácica eram normais. O eletrocardiograma tinha ritmo sinusal. No exame físico, a veia safena magna (VSM) apresentava-se dilatada e com

\footnotetext{
Médico Assistente da Disciplina de Angiologia e Cirurgia Vascular e Endovascular da Faculdade de Medicina do ABC (FMABC) - Santo André (SP), Brasil; Especialista em Cirurgia Vascular, Angiorradiologia e Cirurgia Endovascular pela Sociedade Brasileira de Angiologia e Cirurgia Vascular (SBACV) e pela Associação Médica Brasileira (AMB); Especialista em Radiologia Intervencionista e Angiorradiologia pelo Colégio Brasileiro de Radiologia e Diagnóstico por Imagem (CBR)/SBACV/AMB.

Médico Responsável pela Seção de Ecografia Vascular da Disciplina de Angiologia e Cirurgia Vascular e Endovascular da FMABC - Santo André (SP), Brasil; Especialista em Cirurgia Vascular e Ecografia Vascular com Doppler pela SBACV/AMB.

3Médico Assistente da Disciplina de Angiologia e Cirurgia Vascular e Endovascular da FMABC - Santo André (SP), Brasil; Especialista em Cirurgia Vascular, Angiorradiologia e Cirurgia Endovascular pela SBACV/AMB.

${ }^{4}$ Médico Colaborador da Disciplina de Angiologia e Cirurgia Vascular e Endovascular da FMABC - Santo André (SP), Brasil; Especialista em Cirurgia Vascular pela SBACV/AMB e Ecografia Vascular com Doppler pela SBACV/AMB/CBR.

5 Professor Doutor Titular da Disciplina de Angiologia e Cirurgia Vascular da FMABC, Santo André (SP), Brasil.

Não foram declarados conflitos de interesse associados à publicação deste artigo.

Submetido em: 6.5.2010. Aceito em: 8.12.2010

J Vasc Bras. 2010;9(4):257-260.
} 
pulsatilidade no nível da junção safeno-femoral (JSF) em ambos os membros inferiores (Figura 1). O ultrassom duplex (USD) pré-operatório do MID evidenciava JSF com $15 \mathrm{~mm}$ de diâmetro, dilatação e refluxo em toda a extensão da VSM e veias tributárias varicosas, estando o sistema venoso profundo (SVP) pérvio e competente.

O procedimento foi realizado com o paciente em decúbito dorsal horizontal e aplicando-se um garrote no nível do joelho. A VSM foi puncionada com Jelco $18 \mathrm{G}$ na porção distal da coxa guiada por USD. A microespuma foi preparada pelo método de Tessari por meio da mistura de solução de polidocanol 3\% e ar ambiente na proporção de 1:4 e $8 \mathrm{~mL}$ da solução foi injetada sobre controle ultrassonográfico em tempo real, enquanto o membro era mantido elevado a $45^{\circ}$ e a JSF comprimida por um outro transdutor ultrassonográfico. Após a injeção, o MID permaneceu elevado a $45^{\circ}$ e a JSF foi comprimida por 10 minutos e, em

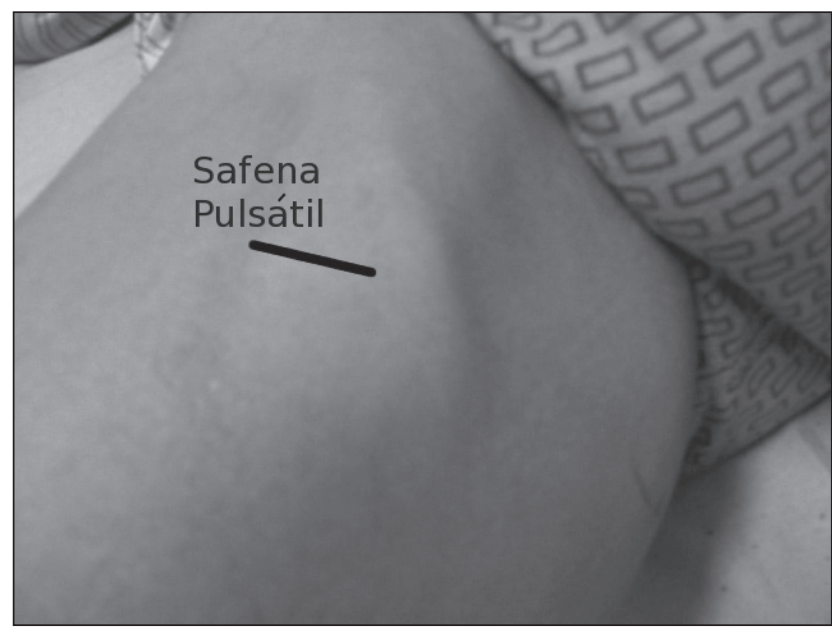

Figura 1 - Veia safena magna dilatada, visível e pulsátil à inspeção clínica na coxa direita

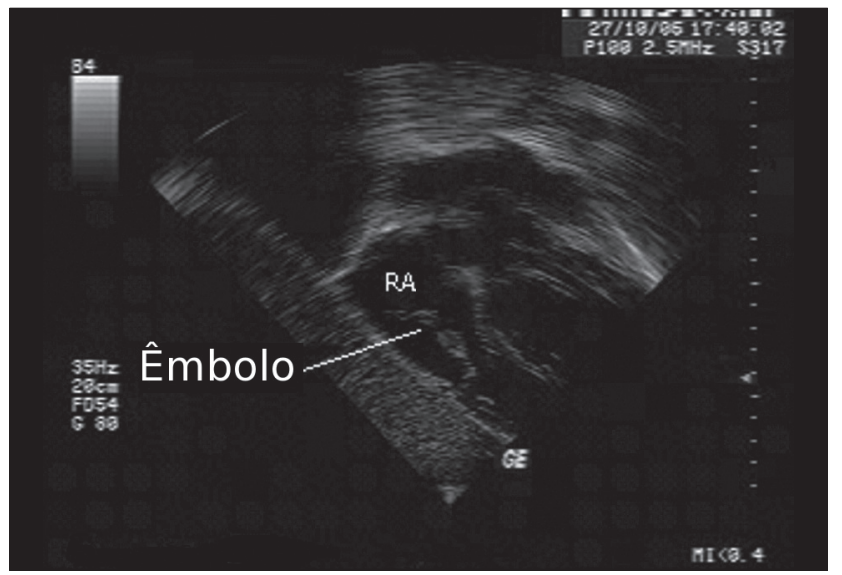

Figura 2 - Ecocardiograma transtorácico mostrando êmbolo móvel no átrio direito seguida, enfaixamento compressivo inelástico foi aplicado e mantido em todo o MID por 24 horas. Posteriormente, prescreveram-se meias elásticas $(30-40 \mathrm{mmHg}$ ) por 30 dias. Não houve intercorrências durante o procedimento.

O paciente retornou sete dias após o procedimento, com evidente melhora clínica subjetiva, e o USD de rotina evidenciou oclusão total da VSM nos dois terços distais da coxa. O segmento proximal da VSM na coxa estava parcialmente ocluído e observou-se a presença de um trombo flutuante na JSF, que se fragmentou durante o exame de USD. Não havia sinais de trombose venosa profunda (TVP). O trombo foi imediatamente identificado ascendendo na veia cava inferior e, em seguida, ecocardiograma transtorácico (ECT) mostrou o êmbolo móvel no átrio direito (Figura 2). O paciente foi posicionado em decúbito lateral esquerdo e encaminhado para a unidade de terapia intensiva. A ausência de trombos na JSF permitiu a ligadura-secção da croça da VSM sob anestesia local, e anticoagulação plena com heparina não-fracionada foi iniciada. Repetiu-se o ECT dois dias depois e o êmbolo não foi mais visto. As gasometrias arteriais foram normais e a tomografia computadorizada helicoidal não mostrou qualquer sinal de embolia pulmonar (EP). O paciente manteve-se completamente assintomático e recebeu alta hospitalar com cumarínicos após cinco dias de observação clínica e controle da anticoagulação. Nenhuma evidência de TVP ou EP foi detectada até seis meses após o procedimento, e a anticoagulação foi suspensa. Consentimento livre e esclarecido do paciente foi obtido para o relato do caso.

\section{Discussão}

A EM é um procedimento terapêutico próspero e que vem revolucionando o manejo da doença venosa varicosa ${ }^{1,2}$. A adoção de sistemas de preparo rápido da microespuma e o auxílio rotineiro do USD aumentaram o interesse pelo método, que pode ser realizado ambulatorialmente sem anestesia em uma ou mais sessões e requer menor período de recuperação do que a cirurgia clássica ${ }^{1,3,4}$. Virtualmente, todos os tipos de veias podem ser tratados, incluindo as situações em que o tratamento cirúrgico convencional tem limitações, como nas classificações funcionais CEAP C4-C5-C6, varizes recorrentes e nos pacientes com elevado risco cirúrgico ${ }^{3,4}$. É crescente o número de publicações acerca do método, porém ainda não há consenso em relação às indicações e contraindicações, variações técnicas e complicações ${ }^{5,6}$.

A eficácia e a segurança do método têm sido ressaltadas em inúmeras publicações ${ }^{1,3,6}$, mas apenas um número 
relativamente pequeno de complicações foram publicadas, quase sempre de pequena gravidade ${ }^{1,2,4}$. Entretanto, a incidência real de complicações é desconhecida ${ }^{2}$, e eventos potencialmente graves e letais já foram descritos, como um caso raro e bem documentado de um acidente vascular encefálico em paciente com forame oval patente de $18 \mathrm{~mm}$ de diâmetro e shunt direito-esquerdo ${ }^{7}$, além de um caso de parada cardiorrespiratória associada à escleroterapia com polidocanol de malformação venosa em paciente pediátrico com síndrome de Klippel-Trenaunay ${ }^{8}$.

O extravazamento do agente esclerosante para o SVP através de veias perfurantes ou da JSF durante o procedimento pode ser responsável por parcela dessas complicações, e meios de evitá-lo são preconizados, como sua compressão digital ou controle ultrassonográfico durante a injeção do esclerosante ${ }^{1,9,10}$. Um estudo prospectivo, controlado e randomizado propôs que a ligadura da JSF sob anestesia local previamente à escleroterapia da VSM aumenta a segurança do procedimento por limitar tal extravasamento ${ }^{11}$. Mais recentemente, Bidwai et al. propuseram a ligadura sob anestesia local das veias perfurantes com clipes após sua localização com USD e controle da JSF com balão de Fogarty ${ }^{12}$.

As complicações maiores tardias são muito raras e representadas quase exclusivamente pelas tromboses, que frequentemente são assintomáticas e diagnosticadas por USD entre 7-14 dias após o procedimento ${ }^{2,4}$. A TVP limita-se mais comumente às veias musculares da panturrilha ou às veias tibiais posteriores. Raramente, ocorre TVP femoral ou poplítea por extensão direta da trombose a partir das junções safeno-femoral/safeno-poplítea ou de veias perfurantes ${ }^{9,10}$. Em um grande registro multicêntrico prospectivo com 12.173 sessões de escleroterapia, foi observado um único caso de TVP femoral e nenhum caso de embolia ${ }^{2}$. Yamaki et al. relataram um caso de TVP com EP sintomática após ligadura alta da VSM associada à escleroterapia com polidocanol ${ }^{13}$.

A compressão do membro após a escleroterapia reduz o refluxo patológico e diâmetro da veia tratada, além de reduzir o risco de $\mathrm{TVP}^{3}$. Ela deve ser iniciada imediatamente após a sessão em todos os pacientes. No entanto, não há consenso sobre os meios compressivos ideais e os períodos em que devem ser aplicados, bem como estudos que confirmem sua indiscutível relevância como complemento da escleroterapia ${ }^{3,5,6}$. No caso aqui apresentado, provavelmente o grande diâmetro da JSF, associado à sua pulsatilidade decorrente da IC, e a dificuldade em mantê-la comprimida após o procedimento tenham contribuído para a não obliteração da VSM e formação de trombo flutuante local. Embora não haja estudos que determinem um diâmetro máximo aceitável da JSF para o tratamento por esse método, esta observação clínica pode ser útil para diminuir a sua indicação nos pacientes com grandes diâmetros da JSF e reduzir eventuais complicações.

\section{Conclusão}

A EM é um tratamento promissor para veias varicosas, já que é simples, facilmente reprodutível, pouco invasivo, custo-efetivo e seguro ${ }^{11}$. Entretanto, pode raramente acompanhar-se de complicações graves, como ilustrado neste caso. Precauções como a seleção criteriosa do paciente, a adequação do agente esclerosante, do sítio de punção e do tipo da veia a ser tratada, a terapia compressiva e a experiência do médico com a escleroterapia líquida, bem como sua capacidade de diagnosticar e tratar eventuais complicações podem contribuir para incrementar a eficácia e a segurança do procedimento ${ }^{3}$.

\section{Referências}

1. Redondo P, Cabrera J. Microfoam sclerotherapy. Semin Cutan Med Surg. 2005;24:175-83.

2. Guex JJ, Allaert FA, Gillet JL, Chleir F. Immediate and midterm complications of sclerotherapy: report of a prospective multicenter registry of 12,173 sclerotherapy sessions. Dermatol Surg. 2005;31:123-28.

3. Zimmet SE. Sclerotherapy treatment of telangiectasias and varicose veins. Tech Vasc Interv Radiol. 2003;6:116-20.

4. Munavalli GS, Weiss RA. Complications of sclerotherapy. Semin Cutan Med Surg. 2007;26:22-8.

5. Breu FX, Guggenbichler S. European Consensus Meeting on Foam Sclerotherapy, April, 4-6, 2003, Tegernsee, Germany. Dermatol Surg. 2004;30:709-17.

6. Rabe E, Pannier-Fischer F, Gerlach H, Breu FX, Guggenbichler S, Zabel M; German Society of Phlebology. Guidelines for sclerotherapy of varicose veins (ICD 10: 183.0, I83.1, I83.2 and 183.9). Dermatol Surg. 2004;30:687-93.

7. Forlee MV, Grouden M, Moore DJ, Shanik G. Stroke after varicose vein foam injection sclerotherapy. J Vasc Surg. 2006;43:162-4.

8. Marrocco-Trischitta MM, Guerrini P, Abeni D, Stillo F. Reversible cardiac arrest after polidocanol sclerotherapy of peripheral venous malformation. Deramtol Surg. 2002;28:153-5.

9. Smith PC. Chronic venous disease treated by ultrasound guided foam sclerotherapy. Eur J Vasc Endovasc Surg. 2006;32: 577-83.

10. O'Hare JL, Earnshaw JJ. The use of foam sclerotherapy for varicose veins: a survey of the members of the Vascular Society of Great Britain and Ireland. Eur J Vasc Endovasc Surg. 2007;34: 232-5.

11. Bountouroglou DG, Azzam M, Kakkos SK, Pathmarajah M, Young P, Geroulakos G. Ultrasound-guided foam sclerotherapy combined with sapheno-femoral ligation compared to surgical treatment of 
varicose veins: early results of a randomised controlled trial. Eur J Vasc Endovasc Surg. 2006;31:93-100.

12. Bidwai A, Beresford T, Dialynas M, Prionidis J, Panayiotopoulos $Y$, Browne TF. Ballon control of the saphenofemoral junction during foam sclerotherapy: proposed innovation. J Vasc Surg. 2007:46:145-7.

13. Yamaki T, Nozaki M, Sasaki K. Acute massive pulmonary embolism following high ligation combined with compression sclerotherapy for varicose veins report of a case. Dermatol Surg. 1999;25:321-5.
Correspondência Rubens Pierry Ferreira Lopes
Avenida Presidente Getúlio Vargas, 2.829 - Bela Vista II CEP: 45996-001 - Teixeira de Freitas (BA), Brasil E-mail: rubenspierry@gmail.com

Contribuições dos autores Concepção e desenho do estudo: CH, RBM e RPFL Análise e interpretação dos dados: RBM e RPFL Coleta de dados: TCHB e RPFL Redação do artigo: RBM, RPFL e TCHB Revisão crítica do texto: RBM e OK Aprovação final do artigo*: RBM, RPFL e OK Análise estatística: não se aplica Responsabilidade geral pelo estudo: RBM, RPFL e OK Informações sobre financiamento: Centro de Estudos Ohannes Kafejian da Faculdade de Medicina do ABC.

*Todos os autores leram e aprovaram a versão final submetida ao J Vasc Bras. 